\title{
Optical frequency tripling with improved suppression and sideband selection
}

\author{
Manoj P. Thakur, ${ }^{1, *}$ Maria C.R. Medeiros, ${ }^{2}$ Paula Laurêncio, ${ }^{2}$ and John E. Mitchell ${ }^{1}$ \\ ${ }^{1}$ Department of Electronic and Electrical Engineering, University College London, Torrington Place, London, \\ WC1E-7JE, UK \\ ${ }^{2}$ CEOT - Center of Electronics Optoelectronics and Telecommunication, University of Algarve, Campus de \\ Gambelas, 8005-132 Faro, Portugal \\ m.thakur@ee.ucl.ac.uk
}

\begin{abstract}
A novel optical dispersion tolerant millimetre-wave radio-overfibre system using optical frequency tripling technique with enhanced and selectable sideband suppression is demonstrated. The implementation utilises cascaded optical modulators to achieve either an optical single sideband (OSSB) or double sideband-suppressed carrier (DSB-SC) signal with high sideband suppression. Our analysis and simulation results indicate that the achievable suppression ratio of this configuration is only limited by other system factors such as optical noise and drifting of the operational conditions. The OSSB transmission system performance is assessed experimentally by the transport of 4 WiMax channels modulating a $10 \mathrm{GHz}$ optical upconverted RF carrier as well as for optical frequency doubling and tripling. The $10 \mathrm{GHz}$ and tripled carrier at $30 \mathrm{GHz}$ are dispersion tolerant resulting both in an average relative constellation error (RCE) of $-28.7 \mathrm{~dB}$ after $40 \mathrm{~km}$ of fibre.

(C)2011 Optical Society of America

OCIS codes: (060.2330) Fiber optics communications; (060.5625) Radio frequency photonics; (190.2620) Harmonic generation and mixing; (190.7220) Upconversion; (350.4010) Microwaves; (060.2360) Fiber optics links and subsystems; (060.4510) Optical communications; (060.5060) Phase modulation.
\end{abstract}

\section{References and links}

1. A. J. Seeds and K. J. Williams, "Microwave photonics," J. Lightwave Technol. 24(12), 4628-4641 (2006).

2. D. Y. Kim, M. Pelusi, Z. Ahmed, D. Novak, H. F. Liu, and Y. Ogawa, "Ultrastable Millimeter-Wave Signal Generation Using Hybrid Modelocking of a Monolithic DBR Laser," Electron. Lett. 31(9), 733-734 (1995).

3. J. J. Oreilly, P. M. Lane, R. Heidemann, and R. Hofstetter, "Optical-Generation of Very Narrow Linewidth Millimeter-Wave Signals," Electron. Lett. 28, 2309-2311 (1992).

4. G. H. Smith, D. Novak, and Z. Ahmed, "Overcoming chromatic-dispersion effects in fiber-wireless systems incorporating external modulators," IEEE Trans. Microwave Theory Tech. 45(8), 1410-1415 (1997).

5. M. Weiß, M. Huchard, A. Stöhr, B. Charbonnier, S. Fedderwitz, and D. S. Jäger, "60-GHz Photonic MillimeterWave Link for Short- to Medium-Range Wireless Transmission Up to $12.5 \mathrm{~Gb} / \mathrm{s}$," J. Lightwave Technol. 26(15), 2424-2429 (2008).

6. H. C. Chien, A. Chowdhury, Z. Jia, Y. T. Hsueh, and G. K. Chang, "60 GHz millimeter-wave gigabit wireless services over long-reach passive optical network using remote signal regeneration and upconversion," Opt. Express 17(5), 3016-3024 (2009).

7. M. Mohamed, X. Zhang, B. Hraimel, and K. Wu, "Analysis of frequency quadrupling using a single MachZehnder modulator for millimeter-wave generation and distribution over fiber systems," Opt. Express 16(14), 10786-10802 (2008).

8. J. X. Ma, X. J. Xin, J. Yu, C. X. Yu, K. R. Wang, H. Y. Huang, and L. Rao, "Optical millimeter wave generated by octupling the frequency of the local oscillator," J. Opt. Netw. 7(10), 837-845 (2008).

9. M. Mohamed, X. Zhang, B. Hraimel, and K. Wu, "Analysis of frequency quadrupling using a single MachZehnder modulator for millimeter-wave generation and distribution over fiber systems," Opt. Express 16(14), 10786-10802 (2008).

10. C. T. Lin, J. Chen, S. P. Dai, P. C. Peng, and S. Chi, "Impact of Nonlinear Transfer Function and Imperfect Splitting Ratio of MZM on Optical Up-Conversion Employing Double Sideband With Carrier Suppression Modulation," J. Lightwave Technol. 26(15), 2449-2459 (2008).

11. P. Laurêncio, H. Vargues, I. Fortes, R. Avo, and M. C. R. Medeiros, "Dispersion Robustness of Millimeter Waves Generated by Up-Conversion Strategies," Fiber Integr. Opt. 29(6), 441-452 (2010). 
12. R. Avo, P. Laurencio, and M. C. R. Medeiros, "Comparative Study of Optical Up-Conversion Schemes," Icton: 2011 13th International Conference on Transparent Optical Networks, 1-4 (2011).

13. T. Kawanishi, T. Sakamoto, M. Tsuchiya, M. Izutsu, S. Mori, and K. Higuma, "70dB extinction-ratio LiNbO3 optical intensity modulator for two-tone lightwave generation," Optical Fiber Communication Conference/National Fiber Optic Engineers Conference, Vols 1-6, 443-445 (2006).

14. Y. Ogiso, Y. Tsuchiya, S. Shinada, S. Nakajima, T. Kawanishi, and H. Nakajima, "High Extinction-Ratio Integrated Mach-Zehnder Modulator With Active Y-Branch for Optical SSB Signal Generation," IEEE Photon. Tech Lett. 22(12), 941-943 (2010).

15. C. T. Lin, P. T. Shih, J. Chen, W. Jiang, S. P. Dai, P. C. Peng, Y. L. Ho, and S. Chi, "Optical Millimeter-Wave Up-Conversion Employing Frequency Quadrupling Without Optical Filtering," IEEE Trans. Microwave Theory Tech. 57(8), 2084-2092 (2009).

16. G. de Valicourt, M. A. Violas, D. Wake, F. van Dijk, C. Ware, A. Enard, D. Maké, Z. Liu, M. Lamponi, G.-H. Duan, and R. Brenot, "Radio-Over-Fiber Access Network Architecture Based on New Optimized RSOA Devices With Large Modulation Bandwidth and High Linearity," IEEE Trans. Microwave Theory Tech. 58(11), 3248-3258 (2010).

17. B. Davies and J. Conradi, "Hybrid modulator structures for subcarrier and harmonic subcarrier optical single sideband," IEEE Photon. Tech Lett. 10(4), 600-602 (1998).

18. D. Pareit, V. Petrov, B. Lannoo, E. Tanghe, W. Joseph, I. Moerman, P. Demeester, and L. Martens, "A Throughput Analysis at the MAC Layer of Mobile WiMAX," IEEE Wireless Communications and Networking Conference, 1-6 (2010).

19. R. T. Logan, Jnr., "All-optical heterodyne RF signal generation using a mode-locked-laser frequency comb: theory and experiments," IEEE MTT-S Int. Microw. Symp. Dig. 3, 11-16 (2000).

20. S. Jansen, I. Morita, and H. Tanaka, "Carrier-to-signal power ratio in fiber-optic SSB-OFDM transmission systems", IEICE General Conference, Nagoya, Japan, (Institute of Electronics, Information and Communication Engineers, paper B-10-24, (2007).

21. J. James, P. Shen, A. Nkansah, X. Liang, and N. J. Gomes, "Nonlinearity and Noise Effects in Multi-Level Signal Millimeter-Wave Over Fiber Transmission Using Single and Dual Wavelength Modulation," IEEE Trans. Microwave Theory Tech. 58(11), 3189-3198 (2010).

22. L. Xin, H. X. You, G. Ying, L. S. Feng, and Z. M. Shan, "Phase noise floor suppression of the output carrier from double sideband-carrier suppressed modulation system," Sci. China 54, 1312-1320 (2011).

23. J. Armstrong, “OFDM for Optical Communications,” J. Lightwave Technol. 27(3), 189-204 (2009).

\section{Introduction}

The development of millimetre-wave (mm-wave) $(30-300 \mathrm{GHz})$ fibre supported transport networks to enable mobile network backhaul and pico-cellular wireless personal area network (WPAN) architectures with simplified antenna units are a vital component of next generation access networks [1]. For distance of $\ll<1 \mathrm{~km}$ between antenna units, compared to 5-10 km for current systems, simplistic base station architecture and high optical component reuse is necessary for cost effectiveness.

For dispersion penalty mitigation at mm-wave frequencies, techniques such as optical single sideband (OSSB) are used, which typically involve the phase and frequency locking of two optical sources separated by the millimetre wave frequency [2] or self-heterodyne techniques using a single optical source modulated by a Mach-Zehnder modulator (MZM) either in a double sideband suppressed optical carrier (DSB-SC) configuration [3] or in a suppressed optical sideband configuration [4]. In self-heterodyne techniques the mixing of the two completely correlated optical sidebands upon photodetection produce a mm-wave signal with spectral linewidth only limited by the linewidth of the RF signal that drives the MZM. DSB-SC besides providing robustness against chromatic dispersion also acts as a frequency doubler. This last characteristic is particularly important since it allows the use of MZMs with a bandwidth of half of the required frequency of the millimetre-wave signal. DSB-SC upconversion technique has also been extensively investigated $[5,6]$ and various approaches for DSB-SC generation have been evaluated [7] analytically to show carrier suppression up to $53 \mathrm{~dB}$. The technique proposed in this paper shows carrier/sideband suppression higher than $80 \mathrm{~dB}$ (simulated) and 45-50 dB (experimentally demonstrated). This technique is different as it is not only capable of generating both DSB-SC and OSSB but also offers flexibility to use both electrical and/or optical modulation, dependant on the architecture. In combination with the colourless nature of the setup, the proposed technique offers a generic solution suitable for various architectural requirements. Other frequency upconversion techniques that take advantage of the intrinsic nonlinear characteristics of MZMs have been developed, either to enable operation with cheap low bandwidth MZM or to provide multiband operation [8, 9].

\#155758 - \$15.00 USD Received 30 Sep 2011; revised 29 Oct 2011; accepted 2 Nov 2011; published 22 Nov 2011

(C) 2011 OSA

12 December 2011 / Vol. 19, No. 26/ OPTICS EXPRESS B458 
The robustness of self-heterodyne techniques against dispersion is limited by the finite extinction ratio (ER) encountered in commercially available MZMs which is typically 20-35 $\mathrm{dB}$. The effect of ER arising from the imperfect power splitting ratio at the MZ Y-splitter has been considered in [10] and further analysed for unmodulated and modulated RF carriers $[11,12]$. Although, recently high $(50-70 \mathrm{~dB})$ ER specifically designed MZMs incorporating active trimmers have been demonstrated [13,14] to achieve $47 \mathrm{~dB}$ carrier/sideband supression, it is important to show how similar or even better extinction ratios can be achieved using conventional (20-30 dB ER) off the shell components.

The objective of this paper is to demonstrate, that the effect of the finite extinction ratio of the MZMs on OSSB and DSB-SC modulation can be greatly reduced by using a dual drive Mach-Zehnder modulator (DD-MZM) cascaded with a phase modulator (PM). This combination could potentially be fabricated as single integrated device not dissimilar to the device of [15]. It is also demonstrated that such strategy enables efficient OSSB based radio frequency tripling with the capability to simultaneously transport 4 (or more) WiMax channels (or wide-band OFDM channels), with an average transmit relative constellation error (RCE) of $-30 \mathrm{~dB}$. Due to the high extinction ratio achieved it is demonstrated that this optical frequency tripling is robust against dispersion, presenting an RCE degradation of less than $(3 \mathrm{~dB})$ over $40 \mathrm{~km}$ of fibre.

The paper is organized as follows. Section 2 presents the operational principle of the proposed ER compensation scheme and discusses its application to DSB-SC and OSSB modulation. Section 3 presents and discusses the experimental setup. Section 4 provides experimental evidence of successful ER compensation and efficient optical frequency. Finally, Section 5 concludes the paper.

\section{Theory of operation}

Optical millimetre wave generation techniques are required to be wavelength agnostic [15, 16] hence, the use of optical filters to achieve OSSB is not possible. Also, the use of low frequency optical components to generate high-frequency electrical carriers is desirable.

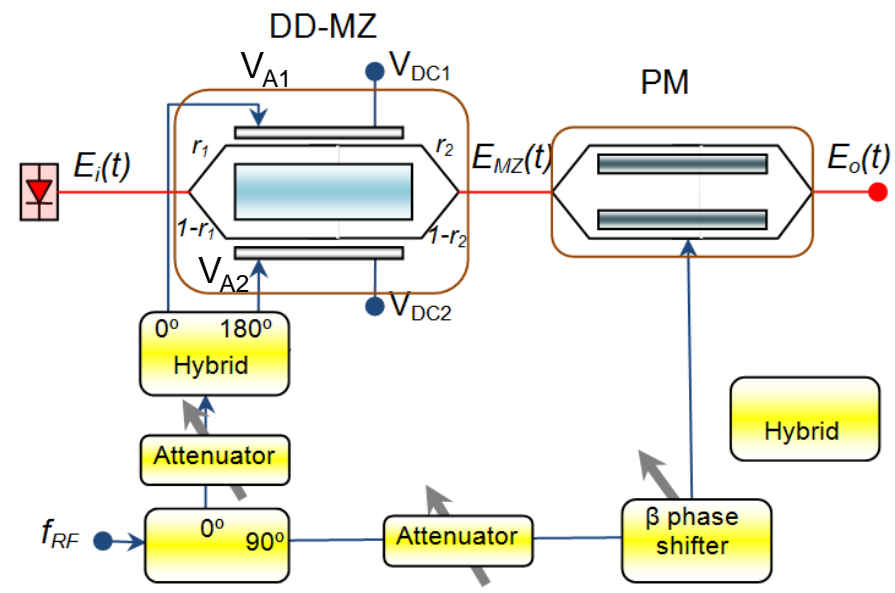

Fig. 1. Schematic of the proposed ER compensation scheme.

The optical modulator used in this work uses a combination of two commercially available components; a DD-MZM cascaded with an optical phase modulator (PM) as illustrated in Fig. 1. The electrical voltages applied on both electrodes of the DD-MZM consist of an RF component at frequency $f_{R F}$ and a dc term, given by:

$$
\begin{aligned}
& V_{A 1}(t)=A \sin \left(2 \pi f_{R F} t\right)-V_{D C 1} \\
& V_{A 2}(t)=-A \sin \left(2 \pi f_{R F} t\right)+V_{D C 2}
\end{aligned}
$$


while the voltage applied to the PM is assumed to be $V_{B}(t)=B \cos \left(2 \pi f_{R F} t+\beta\right), B$ (PM amplitude) and $\beta$ (PM phase) are adjustable parameters. The optical field at the input of the DD-MZM is $E_{i}(t)=\sqrt{2 P_{0}} e^{j \omega_{c} t}$, with optical angular frequency $\omega_{c}$ and average power $P_{0}$. The optical field $E_{M Z}(t)$ at the output of the DD-MZM can be expressed as:

$$
\left.E_{M Z}(t)=E_{i}(t)\left\{\eta \mid e^{j \frac{\pi V_{A 1}(t)}{V_{A \pi}}}+e^{j \frac{\pi V_{A 2}(t)}{V_{A \tau}}}\right\rfloor+\xi e^{j \frac{\pi V_{A 2}(t)}{V_{A \pi}}}\right\}
$$

$V_{A \pi}$ is the switching voltage of the DD-MZM, $\eta$ and $\xi$ are given respectively by $\sqrt{r_{1} r_{2}}$ and $\sqrt{\left(1-r_{1}\right)\left(1-r_{2}\right)}-\sqrt{r_{1} r_{2}}$, where $r_{1}$ and $r_{2}$ are the power splitting ratios of the input and output of the Y-junctions of the MZ [7] respectively. The output of the DD-MZM is therefore modelled as a combination of a balanced DD-MZM with an extra phase modulation. After the phase modulator

$$
E_{o}(t)=E_{i}(t)\left\{\eta\left\lfloor e^{j \frac{\pi V_{A 1}(t)}{V_{A \pi}}}+e^{j \frac{\pi V_{A 2}(t)}{V_{A \pi}}}\right\rfloor+\xi e^{j \frac{\pi V_{A 2}(t)}{V_{A \pi}}}\right\} e^{j \frac{\pi V_{B}(t)}{V_{B \pi}}}
$$

$V_{B \pi}$ being the switching voltage of the phase modulator. Considering $m_{1}=A / V_{A \pi}$ and $m_{2}=B / V_{B \pi}$ the RF modulation indices of the DD-MZM and PM respectively, and $\phi_{1,2}=\frac{\pi V_{D C 1,2}}{V_{A \pi}}$, Eq. (3) can be written after some algebraic manipulations as:

$$
E_{o}(t)=E_{i}(t) \sum_{n=-\infty}^{\infty} j^{n} e^{j n \omega_{R F} t}\left\{\begin{array}{l}
\overbrace{\eta J_{n}\left(\pi M_{1}\right) \cos \left[\frac{n\left(\theta_{1}-\theta_{2}\right)}{2}-\frac{\phi_{1}+\phi_{2}}{2}\right] e^{j\left[\frac{n\left(\theta_{1}+\theta_{2}\right)}{2}-\frac{\phi_{1}-\phi_{2}}{2}\right]}}^{x} \\
+\overbrace{\left[\eta\left(J_{n}\left(\pi M_{2}\right)-J_{n}\left(\pi M_{1}\right)\right)+\xi\right] e^{j\left(n \theta_{2}+\phi_{2}\right)}}^{r}
\end{array}\right\}
$$

where $\theta_{1,2}=\arctan \left(\mp \frac{m_{1}}{m_{2} \cos \beta}+\tan (\beta)\right)$ and $M_{1,2}=\sqrt{m_{1}^{2}+m_{2}^{2} \mp m_{1} m_{2} \sin (\beta)}$.

where $J_{n}($.$) is the Bessel function of the first kind of order n$. The optical field at the output of the PM can then be regarded as a sum of an ideal balanced modulator, with infinite extinction ratio, corresponding to the $X$ term, and extra phase modulation, term $Y$, The extra term, $\eta\left(J_{n}\left(\pi M_{2}\right)-J_{n}\left(\pi M_{1}\right)\right)$, that appears if $\beta \neq 0$, combined with appropriate setting of $m_{l}$ and $m_{2}$ can interact destructively with the term, $\xi$, arising from the MZ imbalance leading to an ER that theoretically can be infinite.

Equation (4) besides explaining and quantifying how the proposed compensation scheme operates also provides a powerful analysis tool that does not require the use of sophisticated simulation software to find out the most appropriated regions of operation. By varying the bias, phase and modulation indices ratios, this configuration provides several interesting features, such as improved side band extinction, as is explored in this paper. Besides OSSB, other modulation modes can be obtained, like the conventional optical DSB-SC at $\phi_{1}-\phi_{2}=\pi$. The flexibility of this configuration allows for fine tuning of the setup for optimized performance and selection of the optical harmonic to be suppressed. The modulation data in turn is present on these components as sidebands. At the receiver, this 
optical signal is square-law detected, and produces frequency components as a result of the beating of the optical signal with its harmonic conjugate. This up-conversion process by optical frequency translation also undergoes phase modulation, which remains unaltered through the nonlinearity of the optical transceiver. Due to this preservation of phase information of the translated RF, the signal can be wirelessly transmitted through the radio link.

\subsection{DSB-SC compensation analysis}

For DSB-SC generation the DD-MZM should be biased with $\phi_{1}=-\phi_{2}=\frac{\pi}{2}$. The output of the DD-MZM is obtained by setting in Eq. (4) $m_{2}=0$ and $\beta=0$, under these circumstances $\theta_{1}=-\theta_{2}=-\frac{\pi}{2}$ and $M_{1}=M_{2}=m_{1}$. The performance of the proposed compensation strategy is assessed, using Eq. (4), in terms of the optical carrier suppression ratio (OCSR), defined as the nower of the first sidehand divided hv the nower of the ontical carrier.

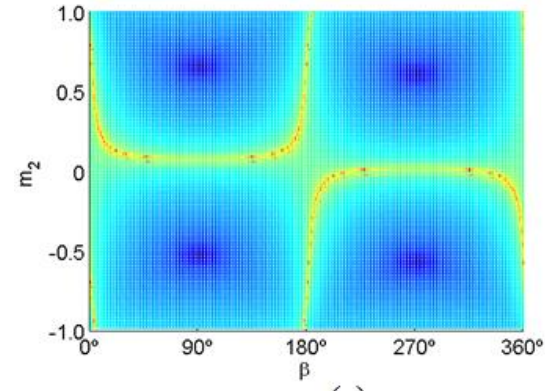

(a)

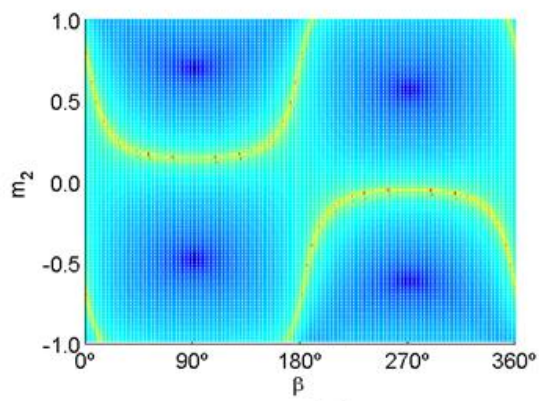

(c)
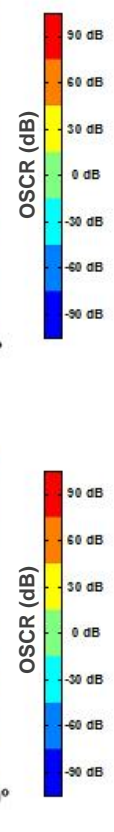

Fig. 2. Dependence of the optical carrier suppression ratio (OCSR) on the modulation index of the phase modulator, $m_{2}$ and the phase shift $\beta$. (a) OCSR contour plot versus $m_{2}$ and $\beta$ for a DD-MZM with ER $=30 \mathrm{~dB}$. (b) Optical power of the optical carrier, first harmonic and second harmonic after ER compensation for the DD-MZM with ER $=30 \mathrm{~dB}$. (c) same as (a) for a DDMZM with $E R=20 \mathrm{~dB}$. (d) Same as (b) for a DD-MZM with ER $=20 \mathrm{~dB}$.

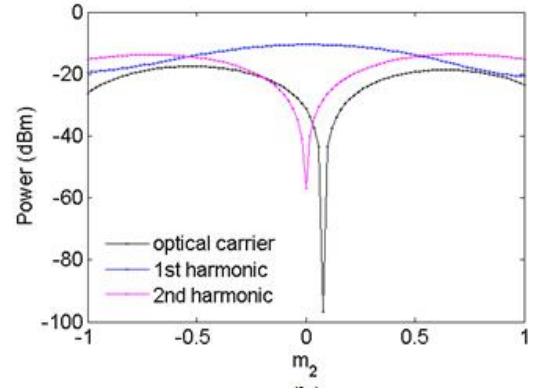

(b)

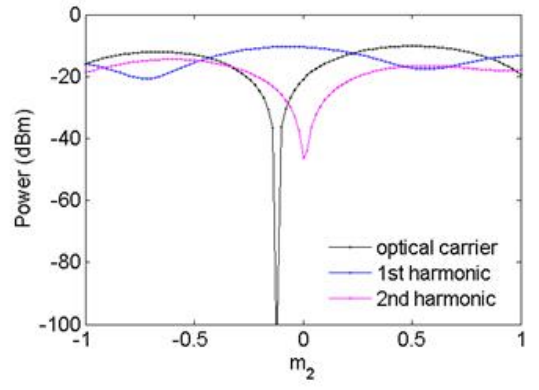

(d) 


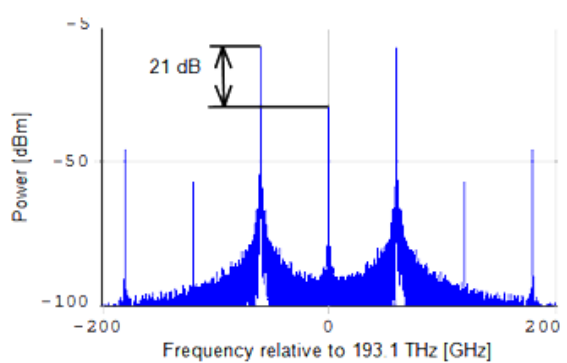

(a)

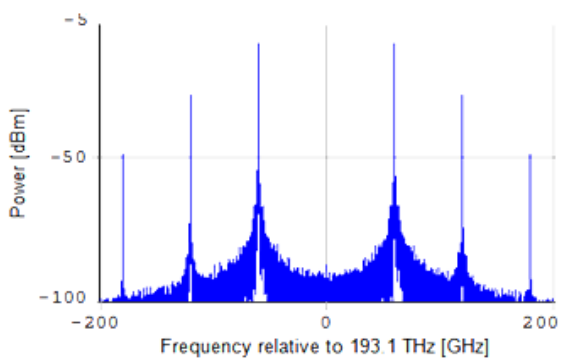

(b)

Fig. 3. Optical spectra, obtained by simulation using the VPI Transmission Maker ${ }^{\mathrm{TM}}$, for $m_{l}=$ $0.2, m_{2}=0.08$ and $\beta=21^{\circ}$. (a) At the output of the DD-MZM. (b) At the output of the PM.

Figure 2(a) and Fig. 2(c) depicts the dependence of the OCSR on the modulation index of the PM, $m_{2}$, and the phase shift $\beta$, in a contour plot. $m_{2}$ is swept in steps of 0.01 while $\beta$ in steps of $1^{\circ}$. Figure 2(a) considers a DD-MZM with an ER $=30 \mathrm{~dB}$ while Fig. 2(c) considers a DD-MZM with ER $=20 \mathrm{~dB}$, the modulation index of the DD-MZM, $m_{l}$, is set to 0.2 . For both situations there are operational regions of improved OCSR, as well as several local maxima values (red colour). Figure 2(b) and Fig. 2(d) show the normalized power of the optical carrier, the first and second optical harmonics at the output of the PM, for $\beta=21^{\circ}$ and $\beta=$ $232^{\circ}$ respectively. These two phase shifts correspond to points of maximum OCSR in the simulation, which in both cases is higher than $80 \mathrm{~dB}$. A word of caution, as can be seen from Fig. 2(a) and Fig. 2(c) the OCSR is very sensitive to $\beta$ and $m_{2}$, therefore a practical implementation of this proposal should employ feedback techniques in order to compensate for operational condition drifts.

Computer simulations were carried out in order to validate our analytical analysis. Figure 3 shows results obtained by simulation using the commercial VPI Transmission Maker software. Figure 3(a) shows the optical spectrum at the output of a DD-MZM with an extinction ratio $\mathrm{ER}=30$ and $m_{l}=0.2$. A CW laser with $1 \mathrm{MHz}$ linewidth and $0 \mathrm{dBm}$ average output power was used in the simulation. After phase compensation, setting $m_{2}=0.08$ and $\beta=$ $21^{\circ}$, as expected from the previous analysis, the optical carrier is highly suppressed, its value being under the system noise level, as shown in Fig. 3(b).

\subsection{OSSB compensation analysis}

Optical single sideband modulation is obtained when $\beta=0, m_{1}=m_{2}$ and $\phi_{1,2}=\pi / 4$, as demonstrated in [17]. In this section, the optical first sideband suppression ratio (OFSSR), defined as the power of the first right sideband divided by the power of the first left sideband is used to evaluate the performance of the proposed compensation technique. For an ideal OSSB signal, OFSSR $= \pm \infty$. The effect of the finite extinction ratio (ER) of the DD-MZM in the optical single side band signal is to generate a spurious right (left) first optical sideband that, if transmission through a dispersive fibre is considered, will interact with the main optical sideband leading to periodic power fading.

OFSSR contour plots are depicted in Fig. 4(a) and Fig. 4(c), versus modulation index of the PM, $m_{2}$, and the phase shift $\beta . m_{2}$ is swept in steps of 0.01 while $\beta$ in steps of $1^{\circ}$. Figure 4(a) considers a DD-MZM with an ER $=30 \mathrm{~dB}$ while Fig. 4(c) considers a DD-MZM with $\mathrm{ER}=20 \mathrm{~dB}$, the modulation index of the DD-MZM, $m_{l}$, is set to 0.2 . Contrary to the compensation of DSB-SC the contour plots show that the optima regions are well defined, and that the optima are reached smoothly. Figure 4(b) and Fig. 4(d) show the normalized power of the optical carrier, the first and second, left and right, optical harmonics at the output of the $\mathrm{PM}$, for $\mathrm{ER}=30 \beta=5^{\circ}$ and $\mathrm{ER}=20, \beta=14^{\circ}$, respectively. In both situations the maximum OFSSR is obtained for $m_{2}=m_{1}=0.2$. The compensation of the ER is accomplished only through an increase in $\beta$. The OSSB configuration is more robust than the OCS configuration and the ER maximum is reached smoothly. 


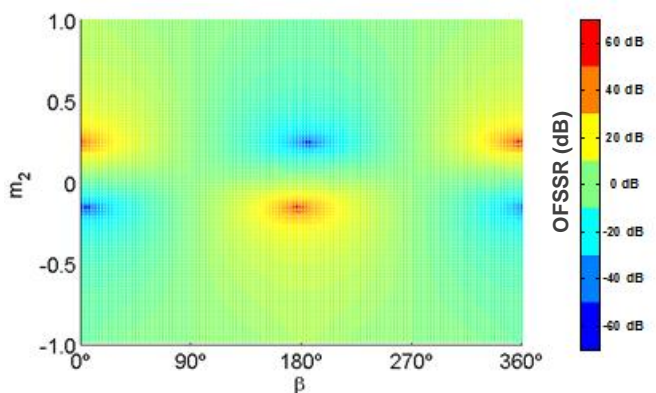

(a)

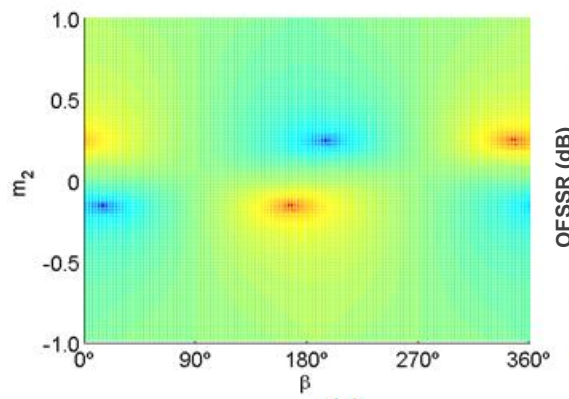

(c)

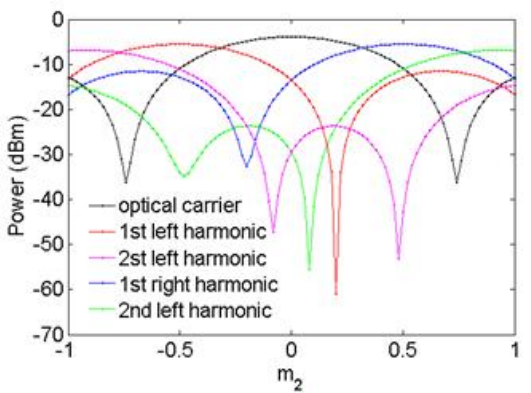

(b)

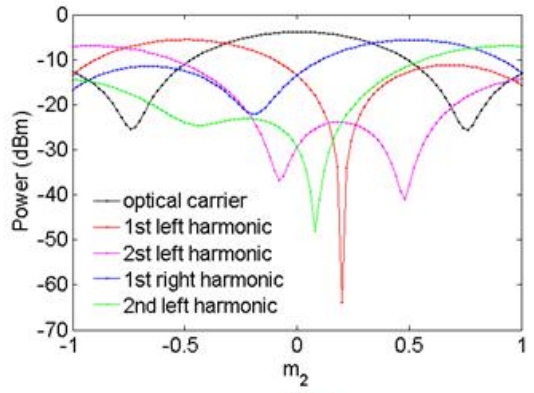

(d)

Fig. 4. Dependence of the OFSSR on the modulation index of the phase modulator, $m_{2}$ and the phase shift $\beta$. (a) OFSSR contour plot versus $m_{2}$ and $\beta$ for a DD-MZM with ER $=30 \mathrm{~dB}$. (b) Optical power of the optical carrier, first harmonics and second harmonics after ER compensation for the DD-MZM with ER $=30 \mathrm{~dB}$. (c) same as (a) for a DD-MZM with ER = $20 \mathrm{~dB}$. (d) Same as (b) for a DD-MZM with $E R=20 \mathrm{~dB}$.

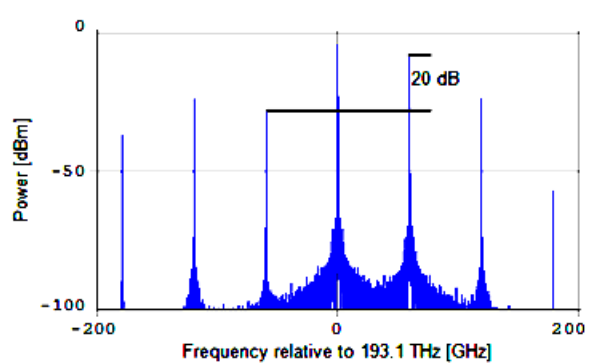

(a)

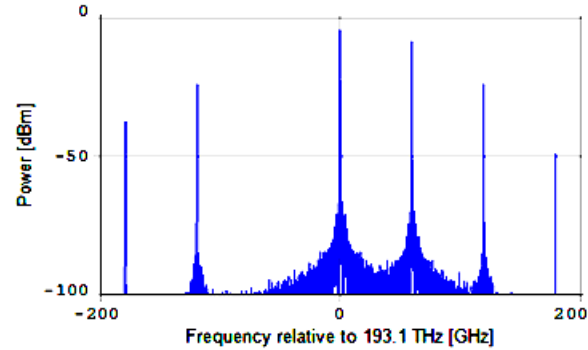

(b)

Fig. 5. OSSB optical spectra, obtained by simulation using the VPI Transmission Maker ${ }^{\mathrm{TM}}$, for $m_{1}=0.2, m_{2}=0.2$ and $\beta=14.24^{\circ}$. (a) At the output of the DD-MZM. (b) At the output of the PM.

Figure 5 shows the results obtained by simulation using the commercial software VPI Transmission Maker. The optical source used in the simulation was a $1 \mathrm{MHz}$ linewidth $\mathrm{CW}$ laser with $0 \mathrm{dBm}$ average output power. The optical spectrum at the output of a DD-MZM with an extinction ratio $E R=20$ and $m_{1}=0.2$ is illustrated in Fig. 5(a). After phase compensation, setting $m_{2}=0.2$ and $\beta=14.24^{\circ}$, the left optical carrier is highly suppressed, its value being under the system noise level, as depicted in Fig. 5(b).

\section{Experimental setup}

In order to demonstrate the operating concept, the test bed shown in Fig. 6 was implemented. An Aeroflex Vector Signal Generator (VSG) was used as WiMax (802.16-2005 specification) transmitter and receiver. Throughput analysis carried out [18] for layer 2 shows that the maximum data rate achievable for the WiMax parameters used in this experiment is $28 \mathrm{Mbps}$. 
This particular profile was selected as it is valid for both fixed and mobile terminals. In the frequency duplex WiMax signal used, a single burst constituted the downstream data region. Four of these channels with the specifications given in Fig. 6, at 440, 460, $480 \& 500 \mathrm{MHz}$ with $20 \mathrm{MHz}$ channel spacing and $10 \mathrm{MHz}$ channel width were generated. The choice of 10 $\mathrm{MHz}$ bandwidth was influenced by the modulation bandwidth of $90 \mathrm{MHz}$ available on the VSG to accommodate 4 channels.

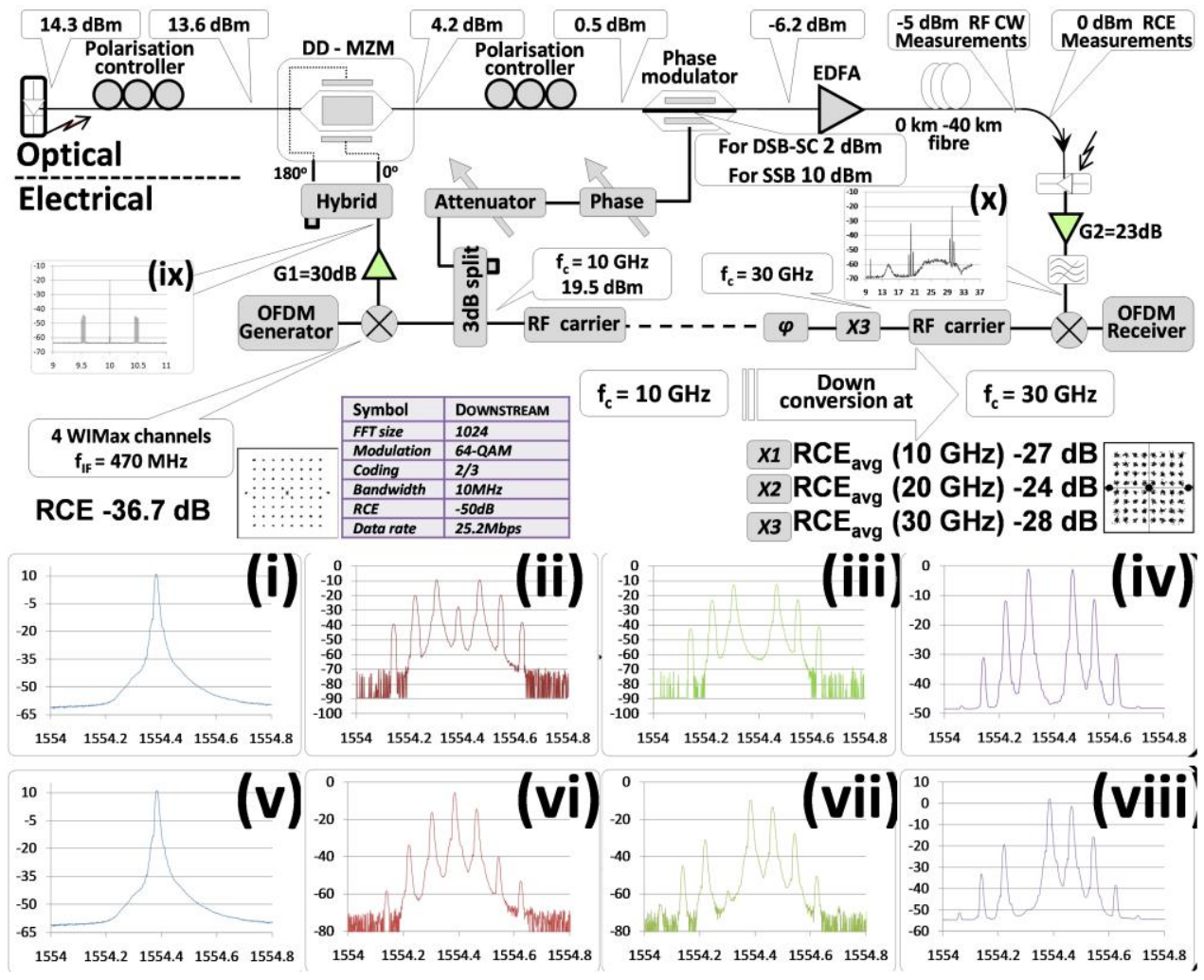

Fig. 6. Cascaded DD-MZM and PM (tuneable to DSB-SC and OSSB configuration)

The channel spacing of $20 \mathrm{MHz}$ was selected to minimise adjacent channel leakage ratio (ACLR) at source. The data rate at source was recorded to be $25.2 \mathrm{Mbps}$ per WiMax channel. The small reduction in the achievable data rate can be attributed to the non-linearity induced penalties in this non-standard WiMax SCM configuration. The back-to-back, baseline downstream WiMax channel transmitter RCE was recorded to be $-36.7 \mathrm{~dB}$. The setup is scalable to wideband signals and higher data rates by using multiple WiMax sources.

To generate useable 3rd order non-linear components, a high RF carrier power level is required at the DD-MZM and the PM inputs. A $19.5 \mathrm{dBm}$ strong $10 \mathrm{GHz}$ RF carrier was split using a $3 \mathrm{~dB}$ splitter with one half fed to a variable electrical attenuator and a variable phase shifter to drive the PM and the other half was mixed with the WiMax signal. The combined electrical power level of the WiMax channels at the input of the mixer was $\square 32 \mathrm{dBm}$. Figure 7 and Fig. 8 show the WiMax signal leakage for transmission measurements. This power level was selected to optimise RCE based on the dynamic range and required modulation index of the system. The up-converted WiMax signal was amplified by a $30 \mathrm{~dB}$ gain amplifier and applied via a $180^{\circ}$ hybrid connected to the arms of DD-MZM, biased at V $\pi=2.65 \mathrm{~V}$. DSB-SC and OSSB with high extinction ratio, as shown in Fig. 6(iv) \& Fig. 6(viii) respectively, were generated by regulating the amplitude and phase of the RF carrier driving the PM. 

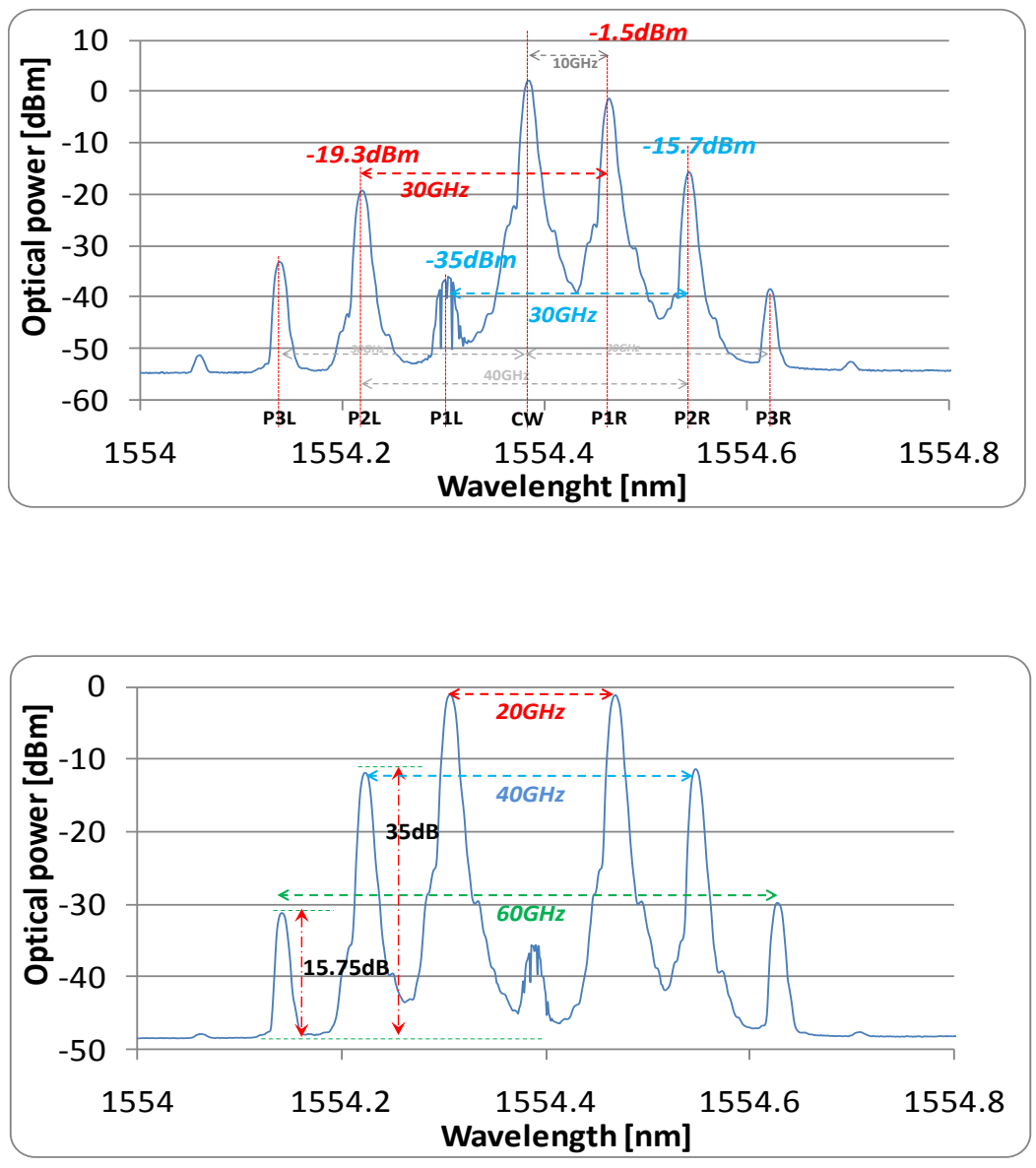

Fig. 8. DSB-SC with modulation data signal leakage

An EDFA was used to maintain $-5 \mathrm{dBm}$ input to a $\mathrm{U}^{2} \mathrm{~T}$ PIN diode with $70 \mathrm{GHz}$ bandwidth and slope efficiency of $0.6 \mathrm{~A} / \mathrm{W}$. After band-pass amplification of the photodetected signal using a Miteq JS4-26004000-25-5P LNA, down-conversion was achieved using a double-balanced mixer. An Aeroflex Vector Signal Analyser was used for demodulation and analysis of the WiMax channels. The SNR of the 4-channel WiMAX signal up-converted to $10 \mathrm{GHz}$ was in excess of $45 \mathrm{~dB}$ (Fig. 6(ix)).

\section{System results and discussion}

For the OSSB setup, measurements evaluated the dispersion induced signal fading for different fibre lengths, shown in Fig. 9. The tripled carrier at $30 \mathrm{GHz}$ was dispersion tolerant. DSB-SC and OSSB configurations with no WiMax data, reached optical carrier and sideband suppressions $>45 \mathrm{~dB}$ (Fig. 6(iii) \& Fig. 6(vii)); a 25dB improvement compared to a typical DD-MZM based OSSB configurations [6-9]. This translates to a lower spurious RF spectrum and higher SNR. Also, the phase imbalance due to asymmetry in the two arms of DD-MZM (when $\mathrm{V}_{\mathrm{DC} 1}=\mathrm{V}_{\mathrm{DC} 2}$ ) can be compensated and used advantageously to fine-tune the resultant phase to either suppress a side-band or the carrier to dynamically switch between OSSB and DSB-SC respectively. The measured optical channel penalty on the SNR of the WiMax signal was $25 \mathrm{~dB}$ (power budget in Fig. 6). Optical modulation index for the DD-MZM was maintained between 0.3 and 0.4 to meet the $-28 \mathrm{~dB}$ RCE requirement to transmit 64 QAM$2 / 3$. Hence the required $18 \mathrm{~dB}$ SNR $($ RCErequired $=-($ SNRrequired +10$))$ was surpassed by $10 \mathrm{~dB}$ as the 


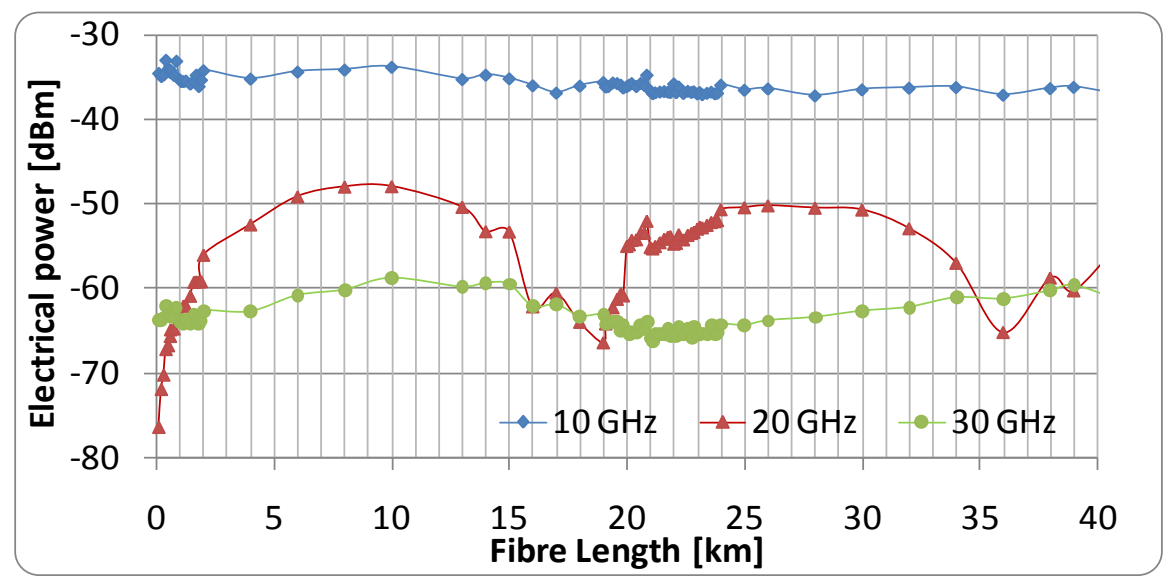

Fig. 9. RF power variation with fibre length for OSSB configuration

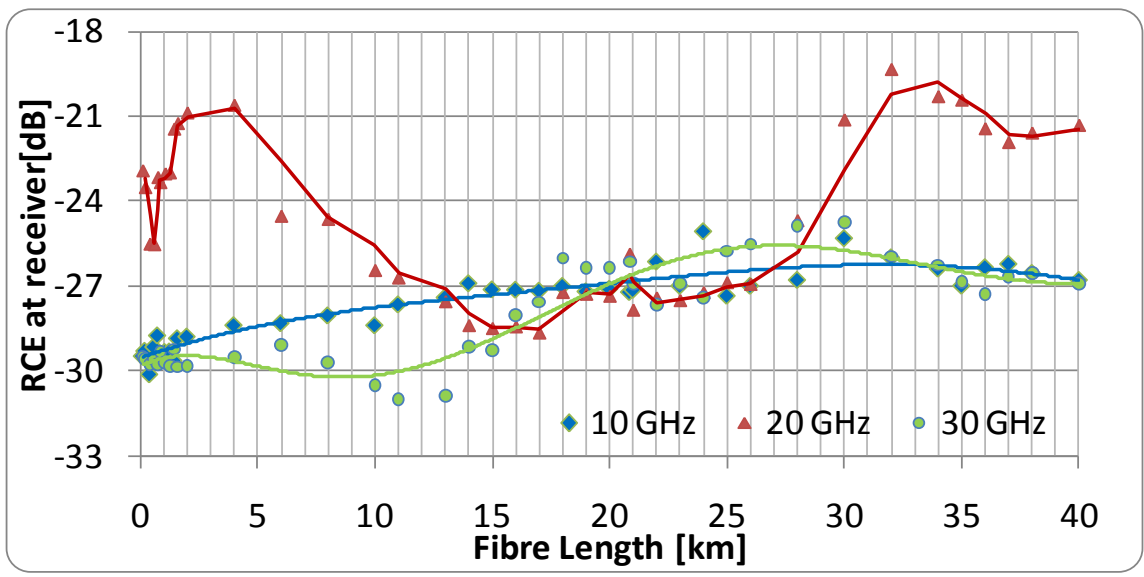

Fig. 10. RCE variation of down-converted WiMax signal.

measured electrical third harmonic (30 GHz) SNR for the system was $27.4 \mathrm{~dB}$. The RCE for $10 \mathrm{GHz}, 20 \mathrm{GHz}$ and $30 \mathrm{GHz}$ were measured for varying fibre lengths and is shown in Fig. 10. For fibre lengths up to $25 \mathrm{~km}$, the demodulated WiMax signal on $30 \mathrm{GHz}$ carrier achieves the $-28 \mathrm{~dB}$ RCE required for $64 \mathrm{QAM}-2 / 3$.

The second harmonic $20 \mathrm{GHz}$ performance is affected by dispersion fading. Sextupling can also be achieved, as previously demonstrated [13] with $25.8 \mathrm{~dB}$ OSNR, in the DSB-SC configuration enabling optical up-conversion to $60 \mathrm{GHz}$. Figure 8 shows only a single pair contribution (dispersion tolerance) to $60 \mathrm{GHz} \mathrm{RF}$ signal. The measured $15.75 \mathrm{~dB}$ relative OSNR for $60 \mathrm{GHz}$ can be improved by using stronger RF carriers. $40 \mathrm{GHz}$ is achievable with this setup with relative OSNR $>35 \mathrm{~dB}$ and carrier suppression $>50 \mathrm{~dB}$, comparable to simulations with $20 \mathrm{GHz}$ carrier and PM.

The phase noise measurement of the upconverted RF signal can be seen in Fig. 11. Table 1 shows the noise values of the RF source and the upconverted signal at various offsets. The measurements show that there is a $9 \mathrm{~dB}$ difference in the phase noise and RF source noise, which is in agreement with the theoretical analysis done [19] to show that phase noise increases as $\mathrm{h}^{2}$ (h being the harmonic multiple, which is 3 in this instance). Walk-off can be observed beyond $100 \mathrm{kHz}$ and can be attributed to relative intensity noise (RIN) of the laser and EDFA noise factor. Also, due to the absence of optical filters and hence higher amplified spontaneous emission (ASE) noise, the power of the modulated OFDM signal and its spectral

\#155758 - \$15.00 USD Received 30 Sep 2011; revised 29 Oct 2011; accepted 2 Nov 2011; published 22 Nov 2011 (C) 2011 OSA 
separation from optical carrier affect the system performance [20, 21]. As a consequence signal-spontaneous beat noise becomes a dominant factor independent of the EDFA gain [22]. Hence, the upconverted signals SNR can be improved by increasing the OFDM signal power vs optical carrier power, the spectral gap between optical carrier and OFDM bands and the gain of EDFA without inducing further penalty in terms of phase noise.

TABLE 1

Source and Upconverted RF Phase Noise

\begin{tabular}{ccc}
\hline \hline Offset $[\mathbf{k H z}]$ & $\mathbf{1 0 ~} \mathbf{~ H H z}[\mathbf{d B c} / \mathbf{H z}]$ & $\mathbf{3 0 ~} \mathbf{~ G H z}[\mathbf{d B c} / \mathbf{H z}]$ \\
\hline 1 & -86.62 & -77.17 \\
10 & -89.94 & -81.25 \\
100 & -111.35 & -91.64 \\
1000 & -126.07 & -89.24 \\
Residual PM & $0.579^{\circ}$ & $3.198^{\circ}$ \\
& & \\
\hline
\end{tabular}

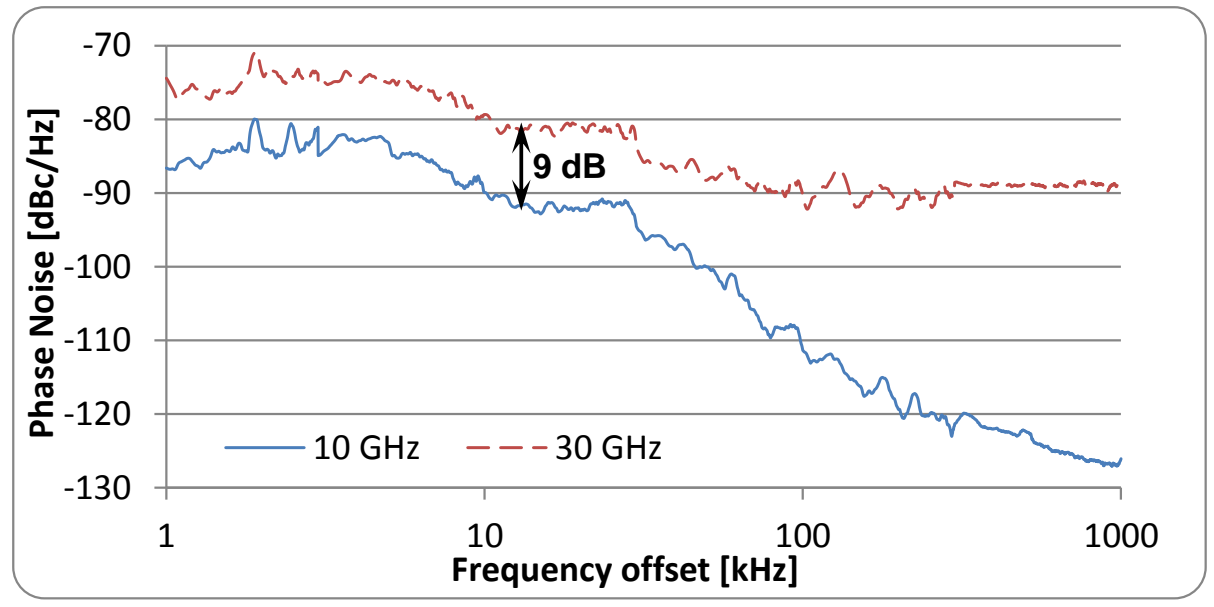

Fig. 11. Measured phase noise of source and upconverted signal.

As for the WiMax signals transmitted, the low phase noise introduced due to tripling of carrier frequency was successfully compensated by the receiver using the pilot tones inherent to OFDM signals [23] and the demodulated WiMax channels constellation can be seen in Fig. 12. Although, an increase in the required modulated signal power and optimum spectral separation from optical carrier has an impact on spectral efficiency, it is easily justified by the colourless nature of the proposed system. 


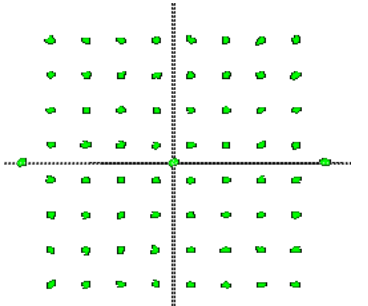

(a)

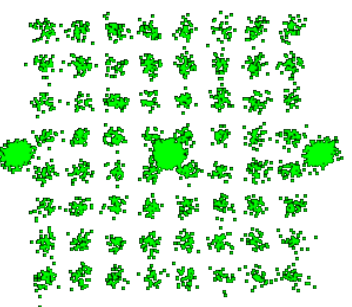

(b)

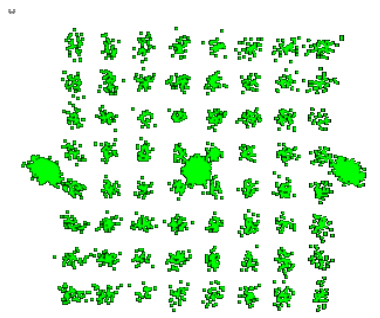

(c)

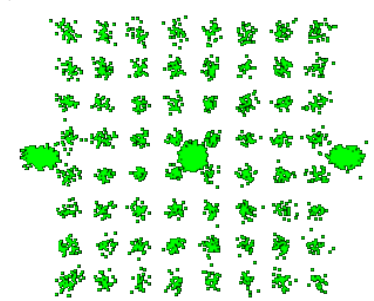

(d)

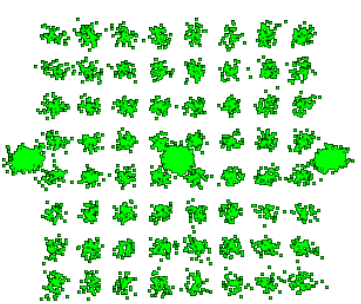

(e)

Fig. 12. Demodulated 64 QAM WiMax signal constellation diagrams obtained after phase and amplitude corrections at the receiver. (a) Electrical back-back. (b) $\mathrm{RF}=30 \mathrm{GHz} \& \mathrm{IF}=440$ MHz. (c) $\mathrm{RF}=30 \mathrm{GHz} \& \mathrm{IF}=460 \mathrm{MHz}$. (d) $\mathrm{RF}=30 \mathrm{GHz} \& \mathrm{IF}=480 \mathrm{MHz}$. (e) $\mathrm{RF}=30 \mathrm{GHz}$ $\& \mathrm{IF}=500 \mathrm{MHz}$.

\section{Conclusion}

In this paper we have reported frequency tripling by employing cascaded MZM \& PM configuration to achieve $>45 \mathrm{~dB}$ sideband suppression.. We have demonstrated theoretically and experimentally that by varying phase and modulation indices ratios of the cascaded configuration, improved side band extinction can be achieved. Moreover, the system has the versatility to select DSB-SC or OSSB operation by varying bias, phase and modulation indices. The concept of multiband system and self-heterodyne reception can be implemented with higher accuracy and lower power. A simple feedback control, monitoring the power level of suppressed optical band can enable automatic optimisation of the bias, phase and attenuation in order to maintain high OSNR and hence the SNR of the overall radio-over-fibre link.

\section{Acknowledgement}

The work described in this paper was carried out with the support of the BONE-project ("Building the Future Optical Network in Europe"), a Network of Excellence funded by the European Commission through the 7th ICT-Framework Programme, and was partially supported by the Portuguese Foundation for Science and Technology within the project PTDC/EEA-TEL/71678/2006 